\title{
Convex Inequalities and Functional Space with the Convex Semi-Norm
}

\author{
Mykola Yaremenko \\ Department Differential Equations \\ National Technical University of Ukraine \\ "Igor Sikorsky Kyiv Polytechnic Institute", Kyiv, \\ Ukraine
}

\begin{abstract}
In this article, we establish new characterizations of convex functions, prove some connected convex type integral inequality; consider the pair of convex functions as the dual semi-norms in functional space. The properties of the integral operators are considered in the scales of the convex semi-norm under the standard conditions on singular kernels.
\end{abstract}

Keywords: - convex, convex function, concave, convex semi-norms, interpolation theorem, singular integral, convex space.

\section{INTRODUCTION}

Convexity is an important mathematical concept of general calculus and functional analysis, especially in applications to the problems of optimization and the consideration of functional spaces. In recent years, new questions pertained to the theory of convexity arise, such in [8] authors established new types of inequalities for the differentiable convex functions in the very specific case of $s$-convexity; in [4], the pattern of the intersection of convex sets in Euclidean space has been studied; [17] deals with the generalization of the Godunov-Levin nonnegative functions.

The goal of this article is to establish new properties of the convex functions and apply them to study the integral operators with the singular kernel $K(x, y)$ that satisfies the standard regularity condition. We show that for these operators the following estimation

$$
\langle\mathscr{M}(|T(f)|)\rangle_{\mu} \leq A\langle\mathscr{M}(|f|)\rangle_{\mu}
$$

holds for arbitrary continuous functions $f \in C_{\mathbf{0}}^{\infty}$ with bounded support. $\Omega \subset X$.

Let $X$ be a metric linear space over $\square$ and

Definition 1. A function $f: \Omega \rightarrow \square$ is called convex on $\Omega$ if

$$
f(\lambda x+(1-\lambda) y) \leq \lambda f(x)+(1-\lambda) f(y)
$$

for all $x, y \in \Omega$ and all $\lambda \in[0,1]$.

If we consider an interval $[a, b]$, this definition of the convexity of the function can be rewritten in the form of the following condition

$$
f(x) \leq \frac{b-x}{b-a} f(a)+\frac{x-a}{b-a} f(b)
$$

or

$$
(b-x) f(a)+(a-b) f(x)+(x-a) f(b) \geq 0
$$

for all $x \in[a, b]$.

From an integral perspective, the convex on $[a, b]$ function can be characterized by the inequality

$$
\frac{1}{y-x} \int_{x}^{y} f(z) d z \leq \frac{1}{2}(f(x)+f(y)),
$$

which holds for all $x, y \in[a, b]$ such that $x<y$.

\section{SOME PROPERTIES OR THE CONVEX FUNCTIONS}

We are going to show that the bounded convex function is always continuous.

Theorem 1. Let $\Omega$ be a compact and a function $f: \Omega \rightarrow \square$ be bounded and convex on $\Omega$ then $f$ is continuous on $\Omega$. 
Proof. Let $x_{0} \in \Omega$, for arbitrary positive $\delta$, we denote $M_{\delta}(x)$ and $m_{\delta}(x)$ the supremum and infimum of the function $f$ on the interval $\left(x_{0}-\delta, x_{0}+\delta\right)$

$M_{\delta}(x)=\sup \left\{f(x): x \in\left(x_{0}-\delta, x_{0}+\delta\right) \cap \Omega\right\}$

$m_{\delta}(x)=\inf \left\{f(x): x \in\left(x_{0}-\delta, x_{0}+\delta\right) \cap \Omega\right\}$.

The functions $M(x)$ and $m(x)$ are defined by the formulae

$$
\begin{gathered}
M(x)=\lim _{\delta \rightarrow \mathbf{0}} M_{\delta}(x) \\
m(x)=\lim _{\delta \rightarrow \mathbf{0}} m_{\delta}(x) .
\end{gathered}
$$

The sequence $\quad\left\{M_{\frac{1}{n}}\left(x_{0}\right), n \in \square\right\}$ converges to $M\left(x_{0}\right)$ then for every $M_{\frac{1}{n}}\left(x_{0}\right)$ there is $x_{n} \in\left(x_{0}-\frac{1}{n}, x_{0}+\frac{1}{n}\right)$ such that

$$
M_{\frac{1}{n}}\left(x_{0}\right)-\frac{1}{n}<f\left(x_{n}\right) \leq M_{\frac{1}{n}}\left(x_{0}\right) .
$$

So, for any $\varepsilon>0$, there is a number $N$ such that for all numbers $n>N$ the both inequalities

$$
\left|x_{n}-x_{0}\right| \leq \varepsilon
$$

and

hold.

$$
\left|f\left(x_{n}\right)-M\left(x_{0}\right)\right| \leq \varepsilon
$$

$$
\begin{gathered}
\text { Let us take the sequence } \\
y_{n}=\frac{1}{1-\lambda} x_{n}-\frac{\lambda}{1-\lambda} x_{0} \text { so } y_{n} \underset{n \rightarrow \infty}{\longrightarrow} x_{0} \text { and } \\
f\left(x_{n}\right)=f\left(\lambda x_{0}+(1-\lambda) y_{n}\right) \leq \\
\leq \lambda f\left(x_{0}\right)+(1-\lambda) f\left(y_{n}\right),
\end{gathered}
$$

let us remark that this formula can be rewritten without the parameter $\lambda$ in the form

$$
\left(x_{\mathbf{0}}-y_{n}\right) f\left(x_{n}\right)+\left(y_{n}-x_{n}\right) f\left(x_{\mathbf{0}}\right)+\left(x_{n}-x_{\mathbf{0}}\right) f\left(y_{n}\right) \geq \mathbf{0}
$$

Let us take arbitrary $\varepsilon>0$ there is small $\delta>0$ such that

$$
M_{\delta}\left(x_{0}\right)<M\left(x_{0}\right)+\varepsilon
$$

thus, there is a number $N$ such that for numbers $n>N$, we have $y_{n} \in\left(x_{0}-\delta, x_{0}+\delta\right)$ so

$$
f\left(x_{n}\right)<\lambda f\left(x_{\mathbf{0}}\right)+(1-\lambda) M\left(x_{\mathbf{0}}\right)+(1-\lambda) \varepsilon
$$

and take a limit as $n \rightarrow \infty$ we obtain

$$
M\left(x_{0}\right)<\lambda f\left(x_{0}\right)+(1-\lambda) M\left(x_{0}\right)+(1-\lambda) \varepsilon .
$$

Now, let us take a limit as $\varepsilon \rightarrow 0$ we have

$$
M\left(x_{0}\right) \leq f\left(x_{0}\right)
$$

so

$$
M\left(x_{\mathbf{0}}\right)=f\left(x_{\mathbf{0}}\right) .
$$

Analogically, let us consider the sequence $\left\{m_{\frac{1}{n}}\left(x_{0}\right), n \in \square\right\}$ that converges to $m\left(x_{0}\right)$. For every $m_{\frac{1}{n}}\left(x_{0}\right)$, we find $x_{n} \in\left(x_{0}-\frac{1}{n}, x_{0}+\frac{1}{n}\right)$ such that

$$
m_{\frac{1}{n}}\left(x_{0}\right) \leq f\left(x_{n}\right)<m_{\frac{1}{n}}\left(x_{0}\right)+\frac{1}{n} .
$$

Let us take the sequence $y_{n}=\frac{1}{1-\lambda} x_{\mathbf{0}}-\frac{\lambda}{1-\lambda} x_{n}$, which $y_{n} \underset{n \rightarrow \infty}{\longrightarrow} x_{\mathbf{0}}$ so we have

$$
\begin{aligned}
& f\left(x_{\mathbf{0}}\right)=f\left(\lambda x_{n}+(1-\lambda) y_{n}\right) \leq \\
& \leq \lambda f\left(x_{n}\right)+(1-\lambda) f\left(y_{n}\right) .
\end{aligned}
$$

For arbitrary, $\varepsilon>0$ there is small $\delta>0$ such that

$$
m\left(x_{0}\right)+\varepsilon<m_{\delta}\left(x_{0}\right)
$$

we take large $N$ such that for numbers $n>N$ we have an estimation

$$
f\left(x_{0}\right)<\lambda f\left(x_{n}\right)+(1-\lambda) M\left(x_{0}\right)+(1-\lambda) \varepsilon .
$$

Let us take a limit as $n \rightarrow \infty$

$$
f\left(x_{\mathbf{0}}\right)<\lambda m\left(x_{\mathbf{0}}\right)+(1-\lambda) M\left(x_{\mathbf{0}}\right)+\varepsilon \text {. }
$$

Taking a limit as $\varepsilon \rightarrow 0$ we are obtaining

$$
f\left(x_{0}\right) \leq m\left(x_{0}\right)
$$

so, we have

$$
m\left(x_{0}\right)=f\left(x_{0}\right)=M\left(x_{0}\right)<\infty .
$$

Let us take an arbitrary $\varepsilon>0$ then there is small $\delta>0$ such that

$$
\begin{array}{r}
m\left(x_{0}\right)-\varepsilon<m_{\delta}\left(x_{0}\right) \leq m\left(x_{0}\right), \\
M\left(x_{0}\right) \leq M_{\delta}\left(x_{0}\right)<M\left(x_{0}\right)+\varepsilon
\end{array}
$$

so, for all $x \in\left(x_{0}-\delta, x_{0}+\delta\right) \cap \Omega$, we have

$$
f\left(x_{0}\right)-\varepsilon \leq f(x) \leq f\left(x_{0}\right)+\varepsilon .
$$

Theorem 1 has been proven.

Let us defined the semi-derivatives of the function. 
Definition 2. Let the function $f$ be defined in a neighborhood of the point $x$. If there is the limit

$$
\mathscr{f}(x)=\lim _{\delta \rightarrow 0} \frac{f(x+\delta)-f(x-\delta)}{2 \delta},
$$

it is called a semi-derivative of the function $f$ in the point $x$.

Definition 3. Let the function $f$ be defined in a neighborhood of the point $x$. If the limit

$$
\mathcal{F o}^{\prime}(x)=\lim _{\delta \rightarrow \mathbf{0}} \frac{f(x+\delta)-2 f(x)+f(x-\delta)}{\delta^{2}}
$$

exists, it is called a second semi-derivative of the function $f$ in the point $x$.

Theorem 2. If the function $f$ is continuous on the interval $[a, b]$ and $f(x) \geq 0$ then the function $f$ is convex. function

Proof. For $\sigma>0$, let us introduce the

$$
\begin{aligned}
& \varphi(x)=f(x)-f(a)- \\
& -\frac{f(b)-f(a)}{b-a}(x-a)+\sigma(x-a)(x-b),
\end{aligned}
$$

which is continuous and satisfies boundary conditions $\varphi(a)=\varphi(b)=0$. It is easy to see that $80^{\prime \prime}(x)=2 \sigma, \quad x \in(a, b)$.

Let us assume that there is a point $x_{0} \in(a, b) \quad$ such that $\varphi\left(x_{0}\right) \geq 0$ from $\varphi(a)=\varphi(b)=0$ follows

$$
\frac{\varphi\left(x_{0}+\delta\right)-2 \varphi\left(x_{0}\right)+\varphi\left(x_{0}-\delta\right)}{\delta^{2}} \leq 0
$$

so, we have $\phi^{\prime \prime}\left(x_{0}\right) \leq 0$, however $\phi^{\prime \prime}\left(x_{0}\right)=2 \sigma$. From this contradiction, we are obtaining that $\varphi(x) \leq 0$ for all $x \in(a, b)$.

For all $x \in(a, b)$, we have the inequality

$$
\begin{aligned}
& f(x) \leq f(a)+ \\
& +\frac{f(b)-f(a)}{b-a}(x-a)-\sigma(x-a)(x-b),
\end{aligned}
$$

next, taking the limit as $\sigma$ approaches zero, we have

$$
(b-a)(f(x)-f(a)) \leq(x-a)(f(b)-f(a))
$$

and assuming $x \in[a, b]$, we are obtaining

$$
(b-x) f(a)+(a-b) f(x)+(x-a) f(b) \geq 0 \text {. }
$$

This proves that function $f$ is convex.

Theorem 3. Let there is $\mathcal{F}^{0}(x)$ a second semi-derivative of a continuous function $f$ defined on $[a, b]$. If $\mathscr{f o}^{\circ}(x)$ is bounded and Lebesgue integrable then the function $f$ can be represented by integral formula

$$
f(x)=\int_{a}^{x} d y \int_{a}^{y} f 0(z) d z+A x+B
$$

The proof of this theorem is fairly straightforward.

Theorem. Assume $f: \Omega \rightarrow \square$ is a twice semi-differentiable then in order function $f$ to convex it is necessary and sufficient that function $f^{\prime}(x) \mathrm{f} 0$ for all $x \in \Omega$.

Proof. This theorem is a consequence of the previous theorem; however, we are going to present its independent proof.

Since $f$ is a convex function we have

$$
f(x+\lambda(y-x)) \leq f(x)+\lambda(f(y)-f(x))
$$

for all $x, y \in \Omega, \quad \lambda \in[0,1]$.

So, for all $\lambda \in[0,1]$, we can write

$$
\lambda(f(y)-f(x)) \geq f(x+\lambda(y-x))-f(x)
$$

and

$$
f(y)-f(x) \geq \mathscr{f o}(x)(y-x)
$$

Similarly, we have

$$
f(x)-f(y) \geq \mathscr{f o}(y)(x-y)
$$

so

$$
f^{\circ}(x)(y-x) \leq f(y)-f(x) \leq f \circ(y)(y-x)
$$

and we deduce

$$
0 \leq \frac{f^{\circ}(y)-\mathscr{f}(x)}{y-x}, \quad x \neq y .
$$

Thus, theorem 3 has been proven.

\section{INTEGRAL PROPERTIES OF THE CONVEX FUNCTION}

Theorem 4. Let the function $f$ be defined and integrable on the interval $[a, b]$, and $f$ satisfies the integral condition

$$
\frac{2}{y-x} \int_{x}^{y} f(z) d z \leq f(x)+f(y)
$$

for all $x, y \in[a, b], \quad x<y$. 
Then the function $f$ is convex on $[a, b]$.

Proof. Assume function $f$ is not convex on $[a, b]$. So, there are $x, y \in[a, b], \quad x<y$ such that

$$
f(\lambda x+(1-\lambda) y)>\lambda f(x)+(1-\lambda) f(y)
$$

for all $\lambda \in(0,1)$.

We have the following inequalities

$$
\begin{aligned}
& \frac{1}{y-x} \int_{x}^{y} f(z) d z=\int_{0}^{1} f(\lambda x+(1-\lambda) y) d \lambda> \\
& >\int_{0}^{1}(\lambda f(x)+(1-\lambda) f(y)) d \lambda= \\
& =\frac{1}{2} f(x)+\frac{1}{2} f(y) .
\end{aligned}
$$

This contradiction is proving that the function $f$ must be convex.

Statement 1. Let the function $f$ be defined and integrable on the interval $[a, b]$, and $f$ satisfies the integral condition

$$
f(x) \leq \frac{1}{2 \delta} \int_{x-\delta}^{x+\delta} f(\lambda) d \lambda
$$

for all $x \in[a, b], \quad \delta>0$.

Then the function $f$ is convex on $[a, b]$.

The proof is obvious.

Next, we are going to study the convex functions as the integrals of monotonous functions.

Theorem 5. Let $\mu$ be an increasing function on $\Omega \subset[a, b]$ then its indefinite integral is a convex function.

$$
M^{\circ}(\tau)=\int_{[a, \tau] \cap \Omega} \mu(t) d t
$$

Proof. Let us assume $\tau_{1}, \tau_{2} \in[a, b] \cap \Omega$ and $\tau_{1}<\tau_{2}$ then

$$
\begin{aligned}
& M \%\left(\tau_{2}\right)-M\left(\lambda \tau_{1}+(1-\lambda) \tau_{2}\right)= \\
& =\int_{\left[\lambda \tau_{1}+(1-\lambda) \tau_{2}, \tau_{2}\right] \cap \Omega} \mu(t) d t \geq \\
& \geq \lambda\left(\tau_{2}-\tau_{1}\right) \mu\left(\lambda \tau_{1}+(1-\lambda) \tau_{2}\right)
\end{aligned}
$$

and

$$
\begin{aligned}
& (1-\lambda)\left(\tau_{2}-\tau_{1}\right) \mu\left(\lambda \tau_{1}+(1-\lambda) \tau_{2}\right) \geq \\
& \geq \int_{\left[\tau_{1}, \lambda \tau_{1}+(1-\lambda) \tau_{2}\right] \cap \Omega} \mu(t) d t= \\
& =M^{\%}\left(\lambda \tau_{1}+(1-\lambda) \tau_{2}\right)-M^{O}\left(\tau_{1}\right)
\end{aligned}
$$

so, we have

$$
\begin{aligned}
& \frac{M^{O}\left(\tau_{2}\right)-M^{\%}\left(\lambda \tau_{1}+(1-\lambda) \tau_{2}\right)}{\lambda} \geq \\
& \geq \frac{M^{\%}\left(\lambda \tau_{1}+(1-\lambda) \tau_{2}\right)-M^{\circ}\left(\tau_{1}\right)}{(1-\lambda)} .
\end{aligned}
$$

Theorem 5 has been proven.

Next, we are going to consider a pair of convex functions as the integrals of monotonous function and its inverse.

Let us assume $\mu(t) \eta(s) t, s \in[0,+\infty)$ are monotonous strictly increasing functions and the function $\eta(s)$ is an inverse to $\mu(t)$ and the function $\mu(t)$ is an inverse to $\eta(s)$ such that

$$
s=\mu(t)=\mu(\eta(s)), \quad \mu(0)=0
$$

and

$$
t=\eta(s)=\eta(\mu(t)), \quad \eta(0)=0 .
$$

We introduce the convex functions

$$
M^{\%}(\tau)=\int_{0}^{\tau} \mu(t) d t \text { and }
$$

$$
\not \mathcal{P}(\tau)=\int_{0}^{\tau} \eta(s) d s, \quad \tau \in[0,+\infty)
$$

There is an integral inequality

$$
\begin{aligned}
& |\langle f, g\rangle| \leq \int_{0}^{\infty} \mu(\lambda) \operatorname{mes}\left\{x \in R^{l}:|f|>\lambda\right\} d \lambda+ \\
& +\int_{0}^{\infty} \mu^{-1}(\lambda) \operatorname{mes}\left\{x \in R^{l}:|g|>\lambda\right\} d \lambda
\end{aligned}
$$

for the arbitrary monotonous strictly increasing function $\mu(t)$ of the real argument $\tau \in[\mathbf{0},+\infty)$.

Let us consider two important exemplars.

1. Holder inequality. Let us presume that

$$
\mu(t)=t^{p-1}, \quad t \in[0,+\infty), \quad p \geq 2
$$

then

so we have

$$
\eta(s)=s^{q}, \quad s \in[0,+\infty), \frac{1}{p}+\frac{1}{q}=1
$$

$$
\mathscr{M}(\tau)=\frac{\tau^{p}}{p}, \quad \tau \in[0,+\infty)
$$

and 


$$
\mathscr{R}(\tau)=\frac{\tau^{q}}{q}, \quad \tau \in[0,+\infty)
$$

Then, we have Holder inequality in the form

$$
a b \leq \frac{a^{p}}{p}+\frac{b^{q}}{q}, \quad \frac{1}{p}+\frac{1}{q}=1 .
$$

2. Exponential function. We take function

$$
\mu(t)=\exp (t)-1, \quad t \in[0,+\infty)
$$

then

$$
\eta(s)=\ln (t+1), \quad t \in[0,+\infty)
$$

we obtain

$$
\mathscr{M}^{\%}(\tau)=\exp (\tau)-\tau-1, \quad \tau \in[0,+\infty)
$$

the complementary function will be

$$
\not f(\tau)=\tau \ln (\tau+1)+\ln (\tau+1)-\tau, \quad \tau \in[0,+\infty) .
$$

Let us assume that $f(x) \equiv f\left(x^{1}, \ldots, x^{l}\right)$ and $g(x) \equiv g\left(x^{1}, \ldots, x^{l}\right), x \in R^{l}, \quad l \in N$ are well define measurable functions such that $\Phi(u) \equiv\langle\mathscr{M}(f)\rangle<\infty$ and $\Upsilon(f) \equiv\langle\mathscr{F}(f)\rangle<\infty$.

Applying properties of Lebesgue integrals, we can write

$$
\langle|f|\rangle=\int_{0}^{\infty} \operatorname{mes}\left\{x \in R^{l}:|f|>\lambda\right\} d \lambda
$$

and

$$
\left\langle M^{\%}(|f|)\right\rangle=\int_{0}^{\infty} \mu(\lambda) \operatorname{mes}\left\{x \in R^{l}:|f|>\lambda\right\} d \lambda .
$$

Theorem 6. Let $f$ be a real positive integrable function. Then we have that

$$
\langle M(f)\rangle_{B} \leq M\left(\langle f\rangle_{B}\right),
$$

where the $B$ is a ball with a measure equal to one.

This theorem can be proven directly as a consequence of Jensen's inequality applied to Lebesgue's sums. We are going to prove this theorem using geometrical arguments, let us rewrite the inequality of the theorem as

$$
\begin{aligned}
& \langle\mathscr{M}(f)\rangle_{B}=\int_{0}^{\infty} \mu(\lambda) \operatorname{mes}\{x \in B: \quad f>\lambda\} d \lambda \leq \\
& \leq M\left(\langle f\rangle_{B}\right)=\int_{0}^{\int_{0}^{\infty} \operatorname{mes}\{x \in B: \quad f>\lambda\} d \lambda} \mu(t) d t .
\end{aligned}
$$

It is easy to see that the Riemann improper integrals here converge so for any natural numbers $n$ and $i$, we can consider a Riemann partition of the real axis $\lambda[0, n]$ as

$$
0=\lambda_{1}<\lambda_{2}<\ldots<\lambda_{i}=n
$$

the Riemann sums are

$$
\begin{aligned}
& \sum_{k} \mu\left(\lambda_{k+1}\right) \operatorname{mes}\left\{x \in B: f>\lambda_{k+1}\right\}\left(\lambda_{k+1}-\lambda_{k}\right) \\
& \text { and }
\end{aligned}
$$

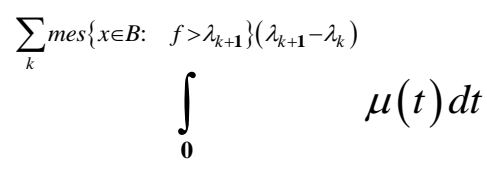

applying strictly monotony of the function $\mu$ we have

$$
\begin{aligned}
& \sum_{k} \mu\left(\lambda_{k+1}\right) \operatorname{mes}\left\{x \in B: \quad f>\lambda_{k+1}\right\}\left(\lambda_{k+1}-\lambda_{k}\right) \leq
\end{aligned}
$$

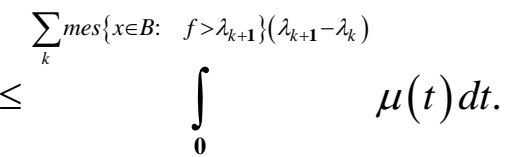

Passing to the limit as $i \rightarrow \infty$ we obtain

$$
\begin{aligned}
& \int_{0}^{n} \mu(\lambda) \text { mes }\{x \in B: \quad f>\lambda\} d \lambda \leq \\
& \leq \int_{0}^{n} \operatorname{mes}\{x \in B: \quad f>\lambda\} d \lambda \\
& \int_{0} \mu(t) d t
\end{aligned}
$$

for any natural numbers $n$. Since these integrals are convergent, we can pass to the limit as $n \rightarrow \infty$ and obtain

$$
\begin{aligned}
& \int_{0}^{\infty} \mu(\lambda) \text { mes }\{x \in B: \quad f>\lambda\} d \lambda \leq \\
& \leq \int_{0}^{\infty} \operatorname{mes}\{x \in B: \quad f>\lambda\} d \lambda \\
& \int_{0} \mu(t) d t .
\end{aligned}
$$

Statement. Since the function $\tilde{M}$ is convex there is the following correlation: let us assume that function $f$ is locally integrable and function $\varphi$ is positive and locally integrable then

$$
M\left(\frac{\int_{a}^{\tau} f(t) \varphi(\tau) d \tau}{\int_{a}^{\tau} \varphi(\tau) \tau}\right) \leq \frac{\int_{a}^{\tau} M^{\%}(f(t)) \varphi(\tau) d \tau}{\int_{a}^{\tau} \varphi(\tau) \tau} .
$$

4. An exemplar of application the convex semi-scale to the integral operator with the singular kernel

Let us consider a class of singular integrals 


$$
\begin{aligned}
& T(f)=\langle K(x-\cdot) f(\cdot)\rangle_{\mu}= \\
& =\int_{R^{l}} K(x-y) f(y) d \mu(y),
\end{aligned}
$$

where the singular kernel is such that this integral is well defined is the sense of distribution and the measurable function-kernel $K(x, y)$ such that

$$
\left|\left(\frac{\partial}{\partial x}\right)^{\alpha} K(x)\right| \leq A|x|^{-l-\alpha}
$$

for all $x \in R^{l}, \quad x \neq 0, \quad|\alpha| \leq 1$.

In accordance with classical theory, the truncated approximations can be defined as

$$
\begin{aligned}
& T_{\varepsilon}(f)=\left\langle K_{\varepsilon}(x-\cdot) f(\cdot)\right\rangle_{\mu}= \\
& =\int_{R^{l}} K_{\varepsilon}(x-y) f(y) d \mu(y),
\end{aligned}
$$

where $\quad K_{\varepsilon}(x)=K(x)$ if $|x| \geq \varepsilon$ and $K_{\varepsilon}(x)=\mathbf{0}$ if $|x|<\varepsilon$. The function $T_{\varepsilon}(f)$ is continuous for all $f \in L^{1}$.

The maximal operator can be defined as

$$
T_{M}(f(x))=\sup _{\varepsilon>\mathbf{0}}\left|T_{\varepsilon}(f(x))\right|
$$

under the condition: there is constant $C$ such that

$$
|T(f(x))| \leq\left|T_{M}(f(x))\right|+C|f(x)| .
$$

Since the

$O=\left\{x: \quad T_{M}(f(x))=\sup _{\varepsilon>\mathbf{0}}\left|T_{\varepsilon}(f(x))\right|>B C\right\} \quad$ is open so we can apply Whitney covering lemma, obtain $O=\bigcup Q_{i}$. Let us consider one of these cubes $\hat{Q}$ with a diameter $d$. According to Whitney covering decomposition, we can find a point $\hat{x} \in{ }^{C} O$ such that $\operatorname{dist}(\hat{x}, O) \leq 4 d$, the ball $B=B(\hat{x}, 6 d)$ so we have $\widehat{Q} \subset B$.

It has to be shown that $\operatorname{mes}\left\{x \in Q: T_{M}(f(x))>\alpha \wedge M(f(x)) \leq c \alpha\right\} \leq$ $\leq \frac{A C}{\mathbf{1}-b} \operatorname{mes} Q$

holds for all cubes

The function $f$ can be presented as the sum $f=f_{1}+f_{2}$, where

$$
f_{1}= \begin{cases}f & x \in B \\ 0 & x \in{ }^{C} B\end{cases}
$$

and

$$
f_{1}= \begin{cases}0 & x \in B \\ f & x \in{ }^{C} B .\end{cases}
$$

So, we have

$$
T_{M}(f) \leq T_{M}\left(f_{1}\right)+T_{M}\left(f_{2}\right)
$$

and

$$
\left\{T_{M}(f)>\alpha\right\} \subset\left\{T_{M}\left(f_{1}\right)>b_{1} \alpha\right\} \cup\left\{T_{M}\left(f_{2}\right)>b_{2} \alpha\right\}
$$

for $b_{1}+b_{2}=1$.

Since, for $f \in L^{1}$, we have

$$
\operatorname{mes}\left\{x \in R^{l}: T_{M} f(x)>\alpha\right\} \leq \frac{A}{\alpha}\langle|f|\rangle
$$

then

$$
\operatorname{mes}\left\{x \in Q: T_{M} f_{1}(x)>\alpha b_{1}\right\} \leq \frac{A}{\alpha b_{1}}\left\langle\left|f_{1}\right|\right\rangle
$$

and

$$
\left\langle\left|f_{1}\right|\right\rangle \leq A \operatorname{Comes}(Q)
$$

so

$$
\operatorname{mes}\left\{x \in Q: T_{M} f_{1}(x)>\alpha b_{1}\right\} \leq \frac{A C}{b_{1}} \operatorname{mes}(Q) \text {. }
$$

If $\quad x \in Q, \quad y \in{ }^{C} B \quad$ and ${ }^{C} B \subset\{y:|y-\hat{y}| \geq d\}$ then

$$
\left|K_{\varepsilon}(\hat{x}-y)-K_{\varepsilon}(x-y)\right| \leq \frac{d A}{|y-\hat{y}|^{l+1}}
$$

and we obtain

$$
\begin{aligned}
& d A\left\langle\frac{|f(\cdot)|}{|\cdot-\hat{y}|^{l+1}}\right\rangle_{|-\hat{y}| \geq d}= \\
= & d A\left\langle\frac{|f(\hat{y}-\cdot)|}{|\cdot|^{l+1}}\right\rangle_{|y| \geq d}= \\
= & \sum_{i} d A\left\langle\frac{|f(\hat{y}-\cdot)|}{|\cdot|^{l+1}}\right\rangle_{2^{i} d \leq|y|<\mathbf{2}^{i+1} d} \leq \\
\leq & \breve{A} \sum_{i} 2^{-i} M(f(\hat{y}))
\end{aligned}
$$

so

$$
\left|T_{M}\left(f_{\mathbf{2}}(\hat{x})\right)-T_{M}\left(f_{1}(x)\right)\right| \leq A M(f(\hat{y}))
$$

for all $x \in Q$. Taking a supremum over $\varepsilon$, we are obtaining

$$
T_{M}\left(f_{2}(x)\right)-T_{M}(f(\hat{x})) \leq A M(f(\hat{y})) \leq \alpha(b+C A)
$$


for $x \in Q$. Assuming that $b_{2} \geq b+C A$, we have that always $T_{M}\left(f_{\mathbf{2}}(x)\right)<\alpha b_{\mathbf{2}}$.

For $b_{1}=\frac{1-b}{2}, b_{2}=1-b, 0<b<1$ and $b_{2} \geq b+C A$, we have

$$
\begin{aligned}
& T(f)=\langle K(x-\cdot) f(\cdot)\rangle_{\mu}= \\
& =\int_{R^{l}} K(x-y) f(y) d \mu(y)
\end{aligned}
$$

where the singular kernel is such that this integral is well defined and the measurable function-kernel for all $\alpha>0$.

Now, we are going to prove that assuming $\omega \in A_{\mathscr{M} /}$ then there is $0<\mathscr{Q} \alpha<1$ such that there is $C>0$

$$
\begin{aligned}
& \omega\left\{x \in R^{l}: T_{M} f(x)>\alpha, M(f(x)) \leq C \alpha\right\} \leq \\
& \leq \mathscr{A} \omega\left\{x \in R^{l}: T_{M} f(x)>\alpha b\right\}
\end{aligned}
$$

holds for $0<b<1$, for all $\alpha>0$.

Indeed, let us take $C$ small enough so that

$\omega\left\{x \in Q: T_{M} f(x)>\alpha, M(f(x)) \leq C \alpha\right\} \leq \breve{C} \omega\{Q\}$

and summing over all cubes we obtain

$$
\begin{aligned}
& \omega\left\{x \in \bigcup Q_{i}: T_{M} f(x)>\alpha, M(f(x)) \leq C \alpha\right\} \leq \\
& \leq \breve{C} \omega\left\{x \in \bigcup Q_{i}: T_{M} f(x)>\alpha b\right\}
\end{aligned}
$$

that proves our statement.

$$
\text { Assuming gives }
$$
$M(f(x)) \geq \frac{c}{(1+|x|)^{n}}$ and for all smooth function, we have inequality

$$
\left|T_{M}(f(x))\right| \leq \frac{A}{(1+|x|)^{n}} .
$$

Every function such that $\langle\tilde{M}(|f| \omega)\rangle<\infty$ can be approximated by elements of $C_{0}^{\infty}$, more precisely, for every function $f,\left\langle M^{\circ}(|f| \omega)\right\rangle<\infty$ and any $\varepsilon>0$ there is a sequence of functions $\phi_{k} \in C_{\mathbf{0}}^{\infty}, \quad k \in N$ and there is a natural number $k_{0}(\varepsilon)$ such that

$$
\left\langle M O\left(\left|f-\phi_{k}\right| \omega\right)\right\rangle<\varepsilon
$$

for every $k>k_{\mathbf{0}}$. The application of this fact concludes the proving of the following theorem.

\section{Theorem 7. Assume that}

for all $x \in R^{l}, \quad x \neq 0, \quad|\alpha| \leq 1$. The maximal operator $T_{M}(f(x))$ satisfies the condition:

$$
|T(f(x))| \leq\left|T_{M}(f(x))\right|+|f(x)| \cdot
$$

\section{Then the integral estimation}

$$
\langle M \%(|T(f)|)\rangle_{\mu} \leq A\langle M \%(|f|)\rangle_{\mu}
$$

holds for all smooth continuous functions $f \in C_{0}^{\infty}$ with bounded support.

\section{REFERENCES}

[1] Acerbi E., Mingione G., Gradient estimates for a class of parabolic systems, Duke Math. J. 136 (2007).

[2] Adimurthi K., Phuc N.C., Global Lorentz, and Lorentz-Morrey estimates below the natural exponent for quasilinear equations, Calc. Var. Partial Differential Equations 54(3) (2015), 3107-3139.

[3] Beni A., Gr"ochenig K., Okoudjou K.A., and Rogers L.G., Unimodular Fourier multiplier for modulation spaces, J. Funct. Anal. 246 (2007), 366-384.

[4] Chen A., Frick F., and Shiu A., Neural codes, decidability, and a new local obstruction to convexity. SIAM Journal on Applied Algebra and Geometry, 3:44-66, (2019).

[5] Chen F. and Liu X., Refinements on the Hermite-Hadamard Inequalities for r-Convex Functions, Journal of Applied Mathematics, vol. 2013, Article ID 978493, 5 pages, 2013.

[6] Guessab, A., Schmeisser G., Sharp integral inequalities of the Hermite-Hadamard type. J. Approx. Theory 115(2), 260-288, (2002).

[7] Iwabuchi T., Navier-Stokes equations and nonlinear heat equations in modulation spaces with negative derivative indices, J. Diff erential Equations 248(8) (2010), 1972-2002.

[8] Kadakal M., Kadakal H., and I. Iscan, Some new integral inequalities for n-times differentiable s-convex functions in the first 
sense," Turkish Journal of Analysis and Number Theory, vol. 5, no. 2, pp. 63-68, 2017.

[9] Noor M.A., Some developments in General Variational inequalities. Appl. Math. Comput., 152, 199-277, (2004).

[10] Ortiz-Caraballo C., Perez C., and Rela E., Exponential decay estimates for singular integral operators, Math. Ann. 357 (2018), 1217-1243.

[11] Wang B., Huo Z., Hao C., Guo Z., Harmonic Analysis Method for Nonlinear Evolution Equations I, Hackensack, NJ: World Scientfic, (2011).

[12] Wang F.Y. Distribution dependent SDEs for Landau type equations. Stochastic Processes and their Applications, 128(2):595-621, 2018.

[13] Xia P., Xie L., Zhang X., and Zhao G. Lq(Lp)theory of stochastic differential equations, (2019).

[14] Zhang X. Stochastic homeomorphism flows of SDEs with singular drifts and Sobolev diffusion coefficients. Electronic Journal of Probability, 16:1096-1116, (2011).

[15] Zhang X. and Zhao G. Singular Brownian Diff usion Processes. Communications in Mathematics and Statistics, 6(4):533-581, (2018).

[16] Zhang X. and Zhao G. Stochastic Lagrangian path for Leray solutions of 3D Navier-Stokes equations, (2019).

[17] Varosanec S., On h -convexity, J. Math. Anal. Appl., 326 (2007), 303-311.

\section{Creative Commons Attribution License 4.0} (Attribution 4.0 International, CC BY 4.0)

This article is published under the terms of the Creative Commons Attribution License 4.0

https://creativecommons.org/licenses/by/4.0/deed.en_US 